\title{
A Simple Neural Network Solar Tracker for Optimizing Conversion Efficiency in Off-Grid Solar Generators
}

\author{
M.A. Panait and T. Tudorache \\ University POLITEHNICA of Bucharest, Electrical Engineering Faculty, Electrical \\ Machines Dept., 313 Splaiul Independentei, 060042, Sect. 6, Bucharest (Romania), \\ Phone/Fax number: +0040 21 3197969, e-mail: axel_morisson@yahoo.com,tudorach@amotion.pub.ro
}

\begin{abstract}
A new model of neural network and a new type of neural controller are proposed, aiming to reduce cost and complexity without sacrificing efficiency of traditional, more complex neural net-based solar trackers.

The solution is derived from Mark Tilden's neural and nervous networks, using a biologic analogy to seamlessly integrate sensors, artificial neurons and effectors in a single, efficient device.

Testing is in progress at the time of the elaboration of this paper but available and relevant preliminary results are shown. The project aims to develop a small pilot tracker - based solar plant for testing purposes and to develop a useable technology for the ever-growing demand for green power.
\end{abstract}

\section{Key words}

Solar tracker, neural networks.

\section{Introduction}

The main element in a solar electric power plant is the solar panel. Physically it consists of a flat surface on which numerous $\mathrm{p}-\mathrm{n}$ junctions are placed, being connected together through electrically conducting strips. As technology evolved, the efficiency of the conversion in solar panels increased steadily, but still it does not exceed $12 \%$ for the most advanced, spherical cell designs. To further complicate matters, the solar panels also exhibit a strongly non-linear I-V characteristic and a power output that is also non-linearly dependant on the surface insolation. The temperature of the panel is also crucial to its normal operation, the silicon junction needing a steady and not too high temperature (80 degrees Celsius being the maximum recommended operating temperature). The optimal performance is attained around 30 degrees Celsius. The dependence of the solar panel performance on the direct insolation is one of the main reasons for a sun tracking system. Compared to a fixed panel, the mobile panel on a tracker is kept under the best possible insolation for all positions of the Sun, as the light falls close to the geometric normal incidence angle.
Solar trackers have been associated with neural networks since the beginning of the study, because as we have seen, the solar panels are strongly non-linear devices and the problem of their output maximization is also a nonlinear problem, the neural networks being well - known for their ability to extract solutions to non-linear problems with variable parameters.

\section{Neural and Nervous Networks}

There are many types of neural networks, some as complicated as multiple redundancy models in the form of complex algorithms running on powerful computers, or as simple as a ring of oscillators having a continuously adjusting duty cycle under the influence of the sensory input. Although research has focused almost exclusively on complicated designs, in the recent years a number of very simple neural networks sprung from the fruitful research of Mark Tilden. He patented two simple configurations that mimic the biological neurons, and several ways to interconnect them [2].

Also from his research, a complementary concept to the well-known neural networks was derived: the nervous network. Following the biological analogy, the nervous network is also a ring of coupled oscillators, much like the central pattern generators in living organisms, controlling the heartbeat or walk gait. These are particularly suitable to be used in conjunction with neural networks to generate motion control sequences, for instance the drive sequences for stepping motors or linear actuators.

The main difference between the classic and this new type of neural controller henceforth referred to as NU-net is that the latter incorporates sensors and actuators as feedback and weight adjusting mechanisms.

Stability and suitability of the NU-net configurations has been proven many times in small autonomous robot configurations as well as in various applications involving intelligent low power motor control.

The main advantages of this configuration over the microprocessor approach is that it needs less power and 
has a much lower cost per unit, without sacrificing the performance.

\section{Paper Main Objectives}

This paper discusses the new generation of neural driven solar trackers, using NU-net and nervous chains (NV-nets) analogues to the structures observed in biologic organisms to achieve a better integration of sensors and effectors, without the need for programmed elements (controllers, memory cells, etc), and presents such a neural controller able to drive a small tracking flat solar panel.

At the end, the final schematic and experimental data gathered to-date are given, along with plans for further improvement.

Solar tracking systems (using light intensity sensing) are shown to boost efficiency of solar conversion up to $100 \%$ during summer and up to $40 \%$ during winter, compared to maximum power point trackers that allow increases of up to $50 \%$ maximum overall.

The final chapter of the work will discuss practical aspects, such as design and technical solutions chosen to satisfy these requirements, and alternatives for scaling the concept to larger plants.

A presentation of the experimental unit is also included.

\section{Solar Tracking and Efficiency}

Solar tracking, like all optimization measures, has some inherent limitations and some parameters to be considered before a final solution is applied. Although beneficial as a method of maximizing solar panel output, tracking is to be made using motors or actuators, and a controller that will add to the "internal service quota" of the solar plant. This has to be carefully balanced to the gains of the system, in each case, if we want to design a completely self-sustaining plant. Still, there are quite a number of research plants implementing several types of solar trackers to compare various solutions and their efficiency, using a power grid connection to provide necessary supply to tracking motors.

The objective of this work is to describe a low power, possibly self-sustaining research solar tracker that uses a rotary encoder to calculate the position of the Sun and to aim several other large panels or instruments that are not mounted on the tracker. Using this system allows for a small panel and a small motor to do the actual tracking, minimizing loss, the angular data being exported to drive the heavy trackers after the position is found. Another requirement for this type of tracker is simplicity in design, a reduced number of moving parts, interchangeability of the main parts and low service quota. That implied using simple solutions based on analog/digital circuitry, to build a new reflex neural-net controller, and small motors and mechanics to keep costs down. An initial version of the tracker, using worm gears was designed, and simulations and calculations showed it to be capable of using two quarter-watt DC or stepping motors (with minimal modifications to the electronic circuit) to drive a small $2.5 \mathrm{~kg}$ solar panel and up to two optical/thermal instruments in tubes, up to $1 \mathrm{~kg}$ each (possibly pyroheliometers or radiometers).

However, it was still expensive as it required machining large diameter gears and a smaller prototype, carrying only sensors, is designed and tested.

The question of efficiency thus is important and crucial even in small scale applications, usually household alternative sources and small plants up to $10 \mathrm{~kW}$.

Considering the high cost-per-watt of the solar energy though, tracking is a viable alternative.

Several reports in USA have shown tracking to be particularly effective in summer, where the increases in output reached $100 \%$, while in autumn they neared 30$40 \%$ depending on the technology used.

Solar tracking implies moving parts, and control systems that tend to be expensive, so single-axis tracking appeared, using a tilted panel mount and a single engine to move the panel on an approximate trajectory relative to the Sun. This system has tracking errors that cannot be corrected and are accepted as such. Still, the efficiency is in all cases of a well designed system, above that of a stationary panel.

Two axis tracking means a specially designed mount able to move the panel on a trajectory as close to the sun path as possible. The errors of this kind of tracker are minimal and can be corrected.

Figures of efficiency boost versus costs vary widely depending on latitude and technological level of the plant, so it is not easy to give accurate figures for a certain installation without complete specifications; however it is accepted that for small deployments, single axis is cheaper while two axis tracking is more efficient, up to $45-50 \%$, according to Red Rock Energy Heliostats (results reported by engineers and independent customers on their solar energy web ring, www.redrok.com). From data presented on various sites and in reports, it is obvious that not only latitude influences the efficiency of tracking, but also the meteorological phenomena and local climate factors, from which the yearly average of the sunny days is the most important [5].

To asses correctly the efficiency of tracking for a certain region, the best way to go is to experiment with a micro plant that has a capacity of 2-3 watts (no more), using both a fixed and a tracking panel, and to constantly monitor the output and compare the efficiency, by automatically calculating the power output over a duration of several months, optimally one year. Such an experiment is currently going on in our university lab, using a data acquisition system to monitor and compute generated power for a fixed and tracking miniature panels. The power consumption from the grid needed to 
power the tracker (small trackers are very difficult to build in a self-sustaining form) is taken into account but it is not subtracted from the total output of the moving panel, because calculations have shown that the relation between the needed motor power, the weight of the panels and their generated power is not linear and does not scale as such, so it would be irrelevant for a larger system.

However it is relevant to compare several tracking solutions, regarding their efficiency at a certain scale (it is expected that for small systems, no tracking and oneaxis tracking to be the optimal solutions while for medium-sized to large systems, two axis tracking to be more effective). This statement is based on data gathered from various sites describing solar technologies and their effectiveness.

\section{The Neural Reflex Network. Basic Topology and Functions}

\section{A. Basic Topology}

The neural controller described in this paper has a lineage directly traceable to Mark Tilden's neural (NU) and nervous (NU) neuron constructs - patent that he kindly released under "Creative Commons "license agreement. The final construct is original only to the extent in which it uses existing parts for a novel function that is to build one of the simplest devices that can control a stepping motor or a dc motor, via a suitable $\mathrm{H}$-bridge, for solar tracking purposes.

The basic topology consists in four independent units (NU neurons), Fig. 1. The basic NU neuron responds to a prolonged change in input. If a high signal is applied to the input, after a delay, the output will switch from high to low and will remain low as long as the input stays high. Because of this behavior, it is suited for slow tracking applications, such as solar tracking.

The basic controller we are going to use in this application features one NV and several NU neurons in a basic configuration, able to drive one stepping motor or one H-bridged DC motor, according to the received input.

The simulations have shown this simple ring oscillator to be self-stabilizing and the green LEDs suitable as sensors, because they provide a very directional response which, although not linear, can be easily approximated with linear curve segments.

\section{B. Functions of the Neural Network}

This particular type of neural network does not work in a classic train-and-replay mode; it does not feature memory whatsoever, apart from short-lived timing interaction between the composing neurons. As such, its functions are more of a reflex network, reacting promptly at environmental changes, monitored by sensors that are directly attached to it.

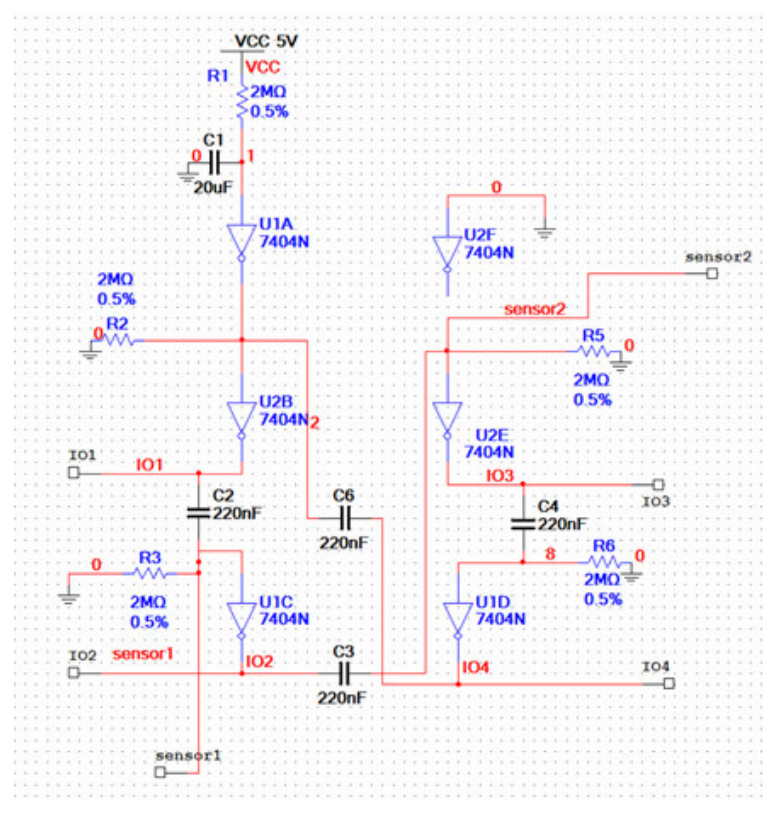

Fig. 1. Tracker core.

The way this tracker works is closer to the phototropic behavior in primitive sea organisms, such as certain species of jellyfish that steer towards the light to keep to the surface. While not so predictable, the output of the network is bounded and self-stabilizing, as it corresponds to limit cycle [1], [4]. The repeatability and stability of the neural network response has been proved by Beiu, Frigo \&Moore in [4].

The transfer function of the neurons is sigmoid, as the classic step output of the logic gates is shaped by the RC low- pass filter. The NV network is thus similar to a nervous net, more than a bona-fide neural net: it responds directly, hard-wired, instead of computing its output. The response time is much faster than that of a classic neural network that requires at least one pass through the entire structure to produce the right output. Here, we have instant response instead, by directly altering the parameters of the circuit. Here the sensors outputs themselves play the role of weights in a classical neural approach, thus we have a very fast, dynamicallyadaptable matrix-shifting neural net, or simpler - a nervous net. The analogy with the reflex path in biological organisms is obvious.

While the network can be active without external input, it needs a time-setting oscillator to set the pace at which the processes take place in the neural net. It has been shown that such a pace-setting oscillator exists in biological organisms and controls, for example, the walking gait the nervous neurons in the net control the walking motions such as position adjustments of the limbs and dynamic equilibrium, but the pace is set by an external oscillator; also the heartbeat is generated by autonomous oscillators, but the pace is set externally.

The external oscillator here injects square pulses into the ring oscillator, and prevents its saturation limiting the oscillation modes to one. 


\section{Tracker Control by Proposed Technique}

The outputs of the nervous neurons are square wave oscillations that are in phase opposition. Their fill factor and their frequency are influenced by the potential at the input nodes, given directly by sensor output. The motor connects at the output nodes, through a reversing $\mathrm{H}$ bridge or buffer amplifier, depending on its configuration, stepping or DC, and is controlled by the pulses to drive the load in a certain direction with a certain speed (if it is a DC motor), or if it has a halfstepping/PWM-controlled stepper driver for a stepping motor.

The light intensity sensors fixed on the panel move as well, and their output changes proportionally (in the case of green LEDs quasi-linearly), and it adjusts the weights to the neuron inputs. Changes in patterns of oscillation take effect immediately.

The research that has been carried out so far was focused on the testing of the proposed neural network technique, on the elaboration of the simulation model of its operation and on the design of the tracker mechanics, Figs. 2 - 5.

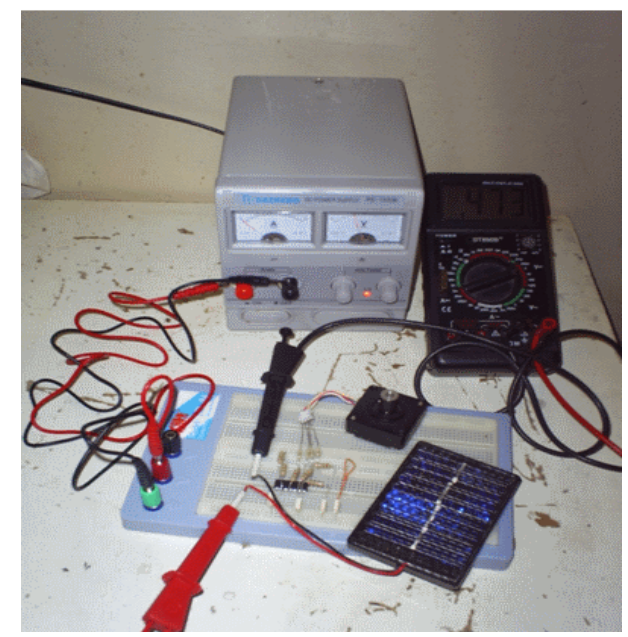

Fig. 2. Testing the proposed Neural Network.

The tracker we have designed is to be used mainly for research, thus it will have the ability of driving a medium-sized (up to 1 sq. $\mathrm{m}$ ) solar panel along with two tube-cased instruments meant to measure the solar radiation and spectral composition of light. The tracker will use incremental encoders to give the absolute position of the Sun and be able to drive an entire array of panels connected via conventional or "slave" neural nets. Simulations made in LabView helped us to design and test neurons prior to their integration in a neural network.

We have found the following function to accurately approximate the output function in any neural-reflex network:

$\mathrm{F}_{\mathrm{n}, \mathrm{a}}(\mathrm{x})=\mathrm{V}_{\mathrm{h}} / 2 \operatorname{sign}[-\sin (2 \pi-(\mathrm{a}-1) \pi / \mathrm{n}) /(\mathrm{T} / \mathrm{rand})]+\mathrm{V}_{\mathrm{h}} / 2$,

where:
- $\quad \mathrm{V}_{\mathrm{h}}=$ oscillation amplitude, given by the Hi level $(5 \mathrm{~V}$ standard for TTL but different, 15 or 3.5 or up to 30 for CMOS, low power CMOS, and industrial logic respectively);

- $\mathrm{a}=$ neuron number in the core, $\mathrm{a}=1,2,3,4$ in this particular $4 \mathrm{NuCore}$

- $\mathrm{n}=$ the number of neurons in the network,

- $\mathrm{T}=$ mean period of oscillation (corresponding to a $50 \%$ fill factor).

This finding helped us to design neural networks having a fundamental frequency suitable to drive stepping motors, avoiding their critical frequency.

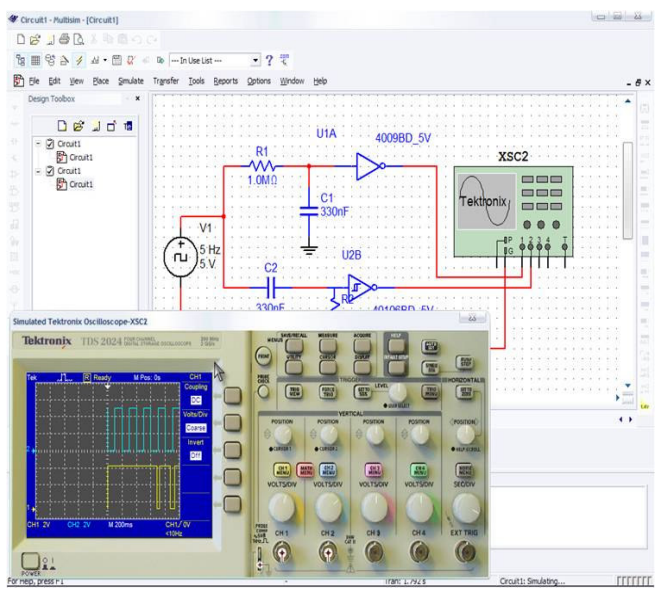

Fig. 3. Simulating the Neural Network parameters

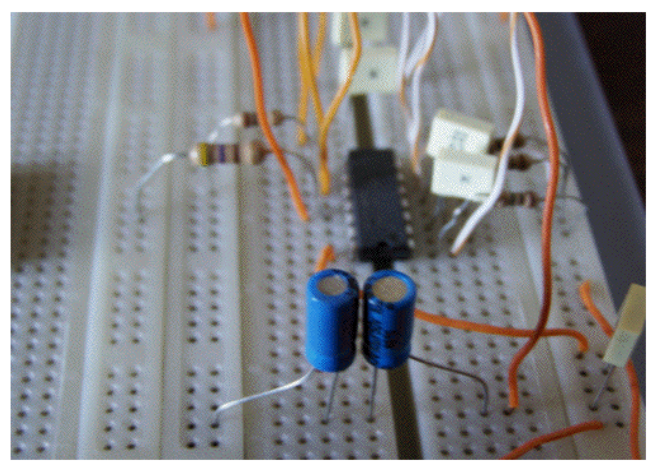

Fig. 4. A close-up of the experimental neural network.

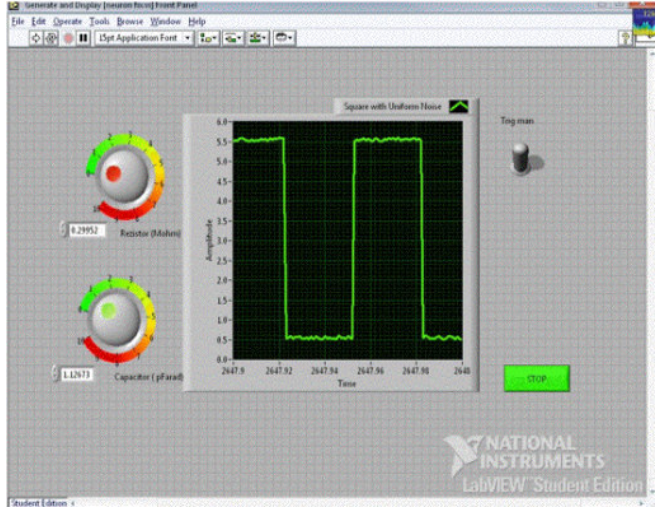

Fig. 5. Using LabView to design neural networks (front panel of a VI showing the capacitor and resistor variable trimmers and the output response). 


\section{Conclusion}

This paper proposes a new model of neural network and a new type of neural controller, aiming to reduce cost and complexity without sacrificing efficiency of traditional, more complex neural net-based solar trackers.

The researches carried out so far are promising being focused on the testing of the proposed neural network technique, on the elaboration of the simulation model of its operation and on the design of the tracker mechanics.

Further researches aim to develop a small pilot tracker based solar plant for testing purposes using the proposed neural network technique.

\section{Acknowledgement}

This paper is a result of the research activity carried out in the frame of two research grants TD 315/2007 funded by CNCSIS and PNII 21-075/2007 funded by CNMP. We take this occasion to thank Romanian projects management institutions CNCSIS and CNMP for their support and also to the Laboratory of Electrical Engineering in Medicine from Univ. POLITEHNICA of Bucharest.

\section{References}

[1] E. A. Rietman and R. W. Hillis, Neural Computation with Rings of Quasiperiodic Oscillators.

[2] B. Hasslacher and M. Tilden, Living Machines, Los Alamos National Laboratory, 2003.

[3] S. Still and M. Tilden, Controller for a four-legged walking machine, 1998.

[4] V. C. Beiu, J. R. Frigo, K. R. Moore, "On the Reliability Of Nervous (Nv) Nets", Computational Intelligence for Modelling Control and Automation, Viena, 12-15 February 1999.

[5] The Red Rock Energy Heliostats webring, www.redrok.com. 